\title{
Narcolepsy associated with primary temporal lobe B-cells lymphoma in a HLA DR2 negative subject
}

\author{
M Onofri, L Curatola, F Ferracci, T Fulgente
}

\begin{abstract}
Narcolepsy and cataplexy began one year before treatment of a left mid-temporal primary B-cells lymphoma in a HLA DR2 negative man. Treatment with radio therapy and immunosuppression induced regression of the lymphoma and disappearance of narcolepsy and cataplexy.
\end{abstract}

$(\mathcal{F}$ Neurol Neurosurg Psychiatry 1992;55:852-853)

Symptomatic or secondary narcolepsy has been described in a number of diseases involving immune system ${ }^{1}$ or in CNS diseases such as Multiple Sclerosis ${ }^{2}$ head trauma, ${ }^{3}$ hypoxic ischaemia ${ }^{4}$ and tumours of the midbrain and diencephalon. ${ }^{5}$ A recent review, however, suggests that that "there is no unequivocal association of narcolepsy with other disorders" 6 and that some cases of "so-called secondary narcolepsy-cataplexy ... may be examples of the association of separate disorders rather than causally related".

The major element prompting the redifinition of secondary narcolepsy was the finding of very high (99\%) narcolepsy association with HLA DR2-DQw1 haplotypes ${ }^{6}$ in idiopathic and secondary cases: only a few white and black DR2 negative patients have been found. $^{78}$

We describe a patient affected by "unequivocal" (according to the criteria suggested by the Association of Sleep Disorders Centers ${ }^{9}$ ) narcolepsy-cataplexy that was secondary to a CNS B-cells lymphoma.

\section{Case report}

The patient, a 30 year old white male, presented to us approximately one year after the onset of excessive daytime sleepiness (EDS) and uncontrollable daytime sleep attacks, lasting from 5 to 10 minutes, preceded by a dramatic abolition of voluntary movement. Such attacks interfered with his daily activities and on one occasion caused a minor driving accident.

In the preceding year his friends had noticed that he was often "dozing-off" and only the complaints of his fiancee forced him to visit our out-patient unit. He sometimes fell to the ground or, if sitting, felt his head dropping without loss of consciousness particularly during exciting or unpleasant situations. The patient was alert and orientated and the neurological examination was normal. Three sets of nocturnal EEGs revealed that "sleep" was spent as follows: $6 \%$ wake, $4 \%$ stage 1 , $46 \%$ stage $2,24 \%$ stage 3,4 and $20 \%$ REM sleep. REM sleep latency was 3.5 minutes. MSLT was carried out in our sleep laboratory after a quiet night's sleep, by means of a polygraph recording EEG, EOG, EMG, ECG and nasal airflow. We recorded 5 scheduled nap periods starting 2 hours after awakening from
Department of

Neurology, Institute of

Neurological and

Behavioural Sciences,

State University of

Chieti, Chieti, Italy

$M$ Onofri

L Curatola

F Ferracci

T Fulgente

Correspondence to:

Dr Onofri, Clinica

Neurologica, Ospedale

ex-Pediatrico, Via Martiri

Lancianesi 6, 66100 Chieti, Italy

Received 13 August 1991 and in final revised form 13 November 1991 . Accepted 20 November 1991

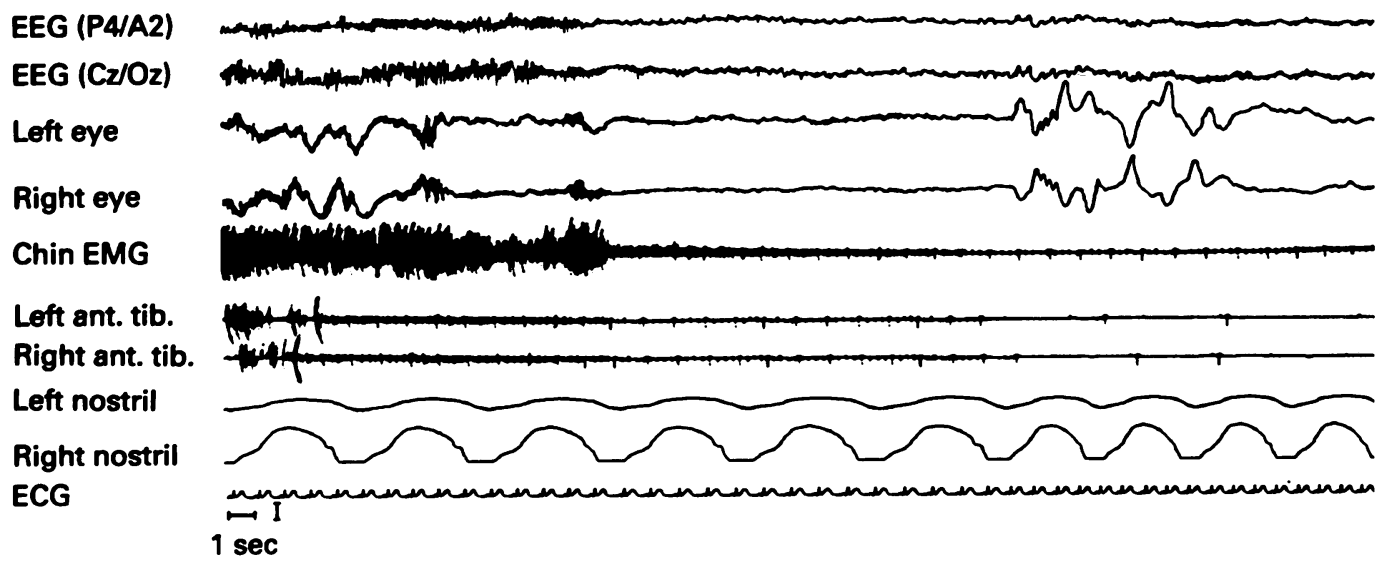

Figure 1 Polygraphic recording, performed one year before the appearance of CNS lymphoma, showing a sleep-onset REM (SOREM) Period. Note the sudden appearance from the stage of wake of a low voltage, fast EEG activity accompanied by Rapid Eye Movements, depression of muscular tone at the EMG and mild increase in heart and respiratory frequency (REM sleep). The monitoring of air flow by nasal thermistors shows no abnormality of breathing and excludes the presence of sleep apneas. Vertical calibration is $50 \mu \mathrm{V}$ for EEG, $100 \mu \mathrm{V}$ for eye movements, $300 \mu \mathrm{V}$ for $E M G$ and $E K G$. 


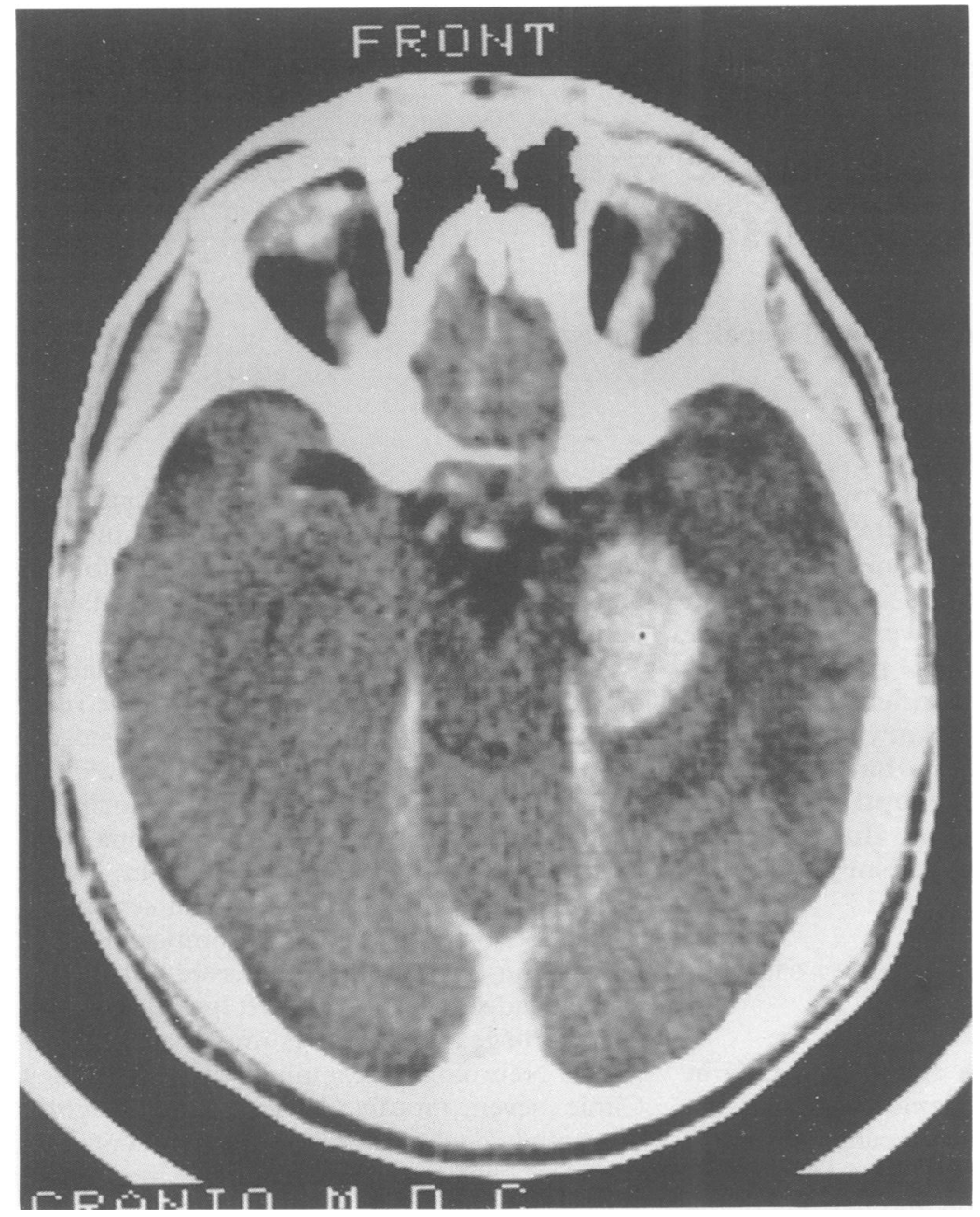

Figure 2 Enhanced CT scan performed one year after the polygraphic recordings in fig 1 revealing a lefi mid-temporal hyperdense area.

nocturnal sleep. Each recording period lasted 15-20 minutes; MSLT was scored on 30 second epochs.

The presence of a mean sleep latency of 40 seconds with 3 sleep onset REM (SOREM) periods on 5 examined naps confirmed the clinical diagnosis of narcolepsy. Figure 1 shows the onset of a SOREM period recorded by polygraphy. CT scan was normal. A brain MRI performed two months later was also normal. The HLA haplotype (by the Trasaki method of microlymphocytotoxicity) was DR4-DQw3. HLA DR and DQ typing in his parents showed that HLA-DR4 was homozygous. HIV antibody testing was negative.

The patient was treated with methylphenidate hydrocloride ( $20 \mathrm{mg}$ three times a day) and tricyclic antidepressants, which improved partially both EDS and sleep attacks. One year after our first observation the patient was admitted with headache, vomiting and stupor without signs of localised brain lesions. CT scan showed a left mid-temporal expansive mass surrounded by an extensive oedematous area (fig 2); MRI confirmed the findings.

A stereotaxic biopsy showed a "primary
CNS non-Hodgkin, high grade, B-cells, centroblastic Lymphoma". HIV antibody testing was negative again. Treatment with prednisone, radiotherapy (5000 cCy) and high dose methotrexate $\left(1 \mathrm{gr} / \mathrm{m}^{2}\right)$ was given. The intracranial hypertension disappeared in weeks. Brain CT performed six months after treatment showed only an area of residual lucency. Nocturnal EEG, and MSLT poligraphic recording, repeated three months, one year and two years after the last CT scan were normal. Narcolepsy and cataplexy were not clinically observed during second admission and in the following 30 months.

\section{Discussion}

After the critical reviews by Bonduelle and Degos $^{10}$ and Parkes ${ }^{6}$ secondary narcolepsy is considered a rare or dubious clinical entity. To our knowledge there are no other reports describing narcolepsy in CNS lymphoma. The course of the disease had furthermore at least three peculiarities differentiating this patient from others reported in the literature: 1) Narcolepsy preceded by one year the appearance of the left mid temporal lobe lymphoma; 2) Narcolepsy disappeared when radiation therapy induced regression of the lymphoma; 3) the patient haplotype was DR2, DQw1 negative.

The typical history of primary brain lymphoma is known to be characterised by behavioural and personality changes or focal neurological signs that precede by several months symptoms due to increased intracranial pressure. We proposed that the unusual clinical picture we describe was due to a focal infiltration of lymphomatous cells in the basal forebrain, in particular the hypothalamus and the area of the third ventricle, which are involved in the regulation of sleep-wake cycle and REM sleep. ${ }^{11}$ Immunotreatment and/or radiotherapy may have suppressed the infiltrat-

ing lymphomatous cells.

1 Langdon N, Lock C, Welsh $\mathrm{K}$, et al. Immune factors in narcolepsy. Sleep 1986;9:143-8.

2 Ekbom K. Familial multiple sclernsis associated with narcolepsy. Arch Neurol 1966;15:337-44.

3 Gill AW. Idiopathic and traumatic Narcolepsy. Lancet 1941;1:474-6.

4 Rivera VM, Meyer JS, Hata T, Iami A. Narcolepsy following cerebral hypoxic ischemia. Ann Neurol 1986;19:505-8.

Aldrich MS, Naylor MW. Narcolepsy associated with lesions of the diencephalon. Neurology 1989;39:1505-9.

6 Parkes JD. Disorders of sleep. In: Bradley WG, et al, eds. Neurology in clinical practice, vol 2. Boston: ButterworthHeinemann 1991:1483-90.

7 Guilleminault C. HLA-DR2 and narcolepsy: not all narcoleptic-cataplectic patients are DR2. Human Immunol 1986;17:1-2.

8 Andreas-Zietz A, Scholz S, Roth B, et al. DR2 negative narcolepsy. Lancet 1986;11:684-5.

9 Association of Sleep Disorders Centers. Diagnostic classification of sleep and arousal disorders. First ed The Sleep cation of sleep and arousal disorders. First ed The Sleep

10 Bonduelle M, Degos C. Symptomatic narcolepsy: a critical study. In Guilleminault C, Dement WC, Passouant P, eds. study. In Guilleminault C, Dement WC, Passou.

11 Jouvet $M$. The regulation of paradoxical sleep by the hypothalamo-hypophysis. Arch Ital Biol 1988;126: 259-74. 